\title{
ON HOPF DEMEYER-KANZAKI GALOIS EXTENSIONS
}

\author{
GEORGE SZETO and LIANYONG XUE
}

Received 15 October 2002

\begin{abstract}
Let $H$ be a finite-dimensional Hopf algebra over a field $k, B$ a left $H$-module algebra, and $H^{*}$ the dual Hopf algebra of $H$. For an $H^{*}$-Azumaya Galois extension $B$ with center $C$, it is shown that $B$ is an $H^{*}$-DeMeyer-Kanzaki Galois extension if and only if $C$ is a maximal commutative separable subalgebra of the smash product $B \# H$. Moreover, the characterization of a commutative Galois algebra as given by S. Ikehata (1981) is generalized.
\end{abstract}

2000 Mathematics Subject Classification: 16W30, 16H05.

1. Introduction. Let $H$ be a finite-dimensional Hopf algebra over a field $k$, $B$ a left $H$-module algebra, and $H^{*}$ the dual Hopf algebra of $H$. In [7], the class of Azumaya Galois extensions of a ring as studied in [1, 2] was generalized to $H^{*}$-Azumaya Galois extensions. An $H^{*}$-Azumaya Galois extension $B$ was characterized in terms of the smash product $B \# H$ see [7, Theorem 3.4]. Observing that the commutator $V_{B}\left(B^{H}\right)$ of $B^{H}$ in $B$ is also an $H^{*}$-Azumaya Galois extension (see [7, Lemma 4.1]), in the present paper, we will give a characterization of an $H^{*}$-Azumaya Galois extension $B$ in terms of $V_{B}\left(B^{H}\right)$. Moreover, we will investigate the class of $H^{*}$-Azumaya Galois extensions $B$ such that $V_{B}\left(B^{H}\right)=C$, where $C$ is the center of $B$. We note that when $H=k G$, where $G$ is a finite automorphism group of $B$, such a $B$ is precisely a DeMeyer-Kanzaki Galois extension with Galois group $G[3,6,8,9]$. Several equivalent conditions are then given for an $H^{*}$-Azumaya Galois extension being an $H^{*}$-DeMeyer-Kanzaki Galois extension, and the characterization of a commutative Galois algebra as given by Ikehata [5, Theorem 2] is generalized to an $H^{*}$-DeMeyer-Kanzaki Galois extension.

2. Basic definitions and notation. Throughout, $H$ denotes a finite-dimensional Hopf algebra over a field $k$ with comultiplication $\Delta$ and counit $\varepsilon, H^{*}$ the dual Hopf algebra of $H, B$ a left $H$-module algebra, $C$ the center of $B, B^{H}=$ $\{b \in B \mid h b=\varepsilon(h) b$ for all $h \in H\}$, and $B \# H$ the smash product of $B$ with $H$, where $B \# H=B \otimes_{k} H$ such that, for all $b \# h$ and $b^{\prime} \# h^{\prime}$ in $B \# H,(b \# h)\left(b^{\prime} \# h^{\prime}\right)=$ $\sum b\left(h_{1} b^{\prime}\right) \# h_{2} h^{\prime}$, where $\Delta(h)=\sum h_{1} \otimes h_{2}$.

For a subring $A$ of $B$ with the same identity 1, we denote the commutator subring of $A$ in $B$ by $V_{B}(A)$. We call $B$ a separable extension of $A$ if there 
exist $\left\{a_{i}, b_{i}\right.$ in $B, i=1,2, \ldots, m$ for some integer $\left.m\right\}$ such that $\sum a_{i} b_{i}=1$ and $\sum b a_{i} \otimes b_{i}=\sum a_{i} \otimes b_{i} b$ for all $b$ in $B$ where $\otimes$ is over $A$. An Azumaya algebra is a separable extension of its center. A ring $B$ is called a Hirata separable extension of $A$ if $B \otimes_{A} B$ is isomorphic to a direct summand of a finite direct sum of $B$ as a $B$-bimodule. A ring $B$ is called an $H^{*}$-Galois extension of $B^{H}$ if $B$ is a right $H^{*}$-comodule algebra with structure map $\rho: B \rightarrow B \otimes_{k} H^{*}$ such that $\beta: B \otimes_{B^{H}} B \rightarrow B \otimes_{k} H^{*}$ is a bijection where $\beta(a \otimes b)=(a \otimes 1) \rho(b)$. An $H^{*}$-Galois extension $B$ is called an $H^{*}$-Azumaya Galois extension if $B$ is separable over $B^{G}$ which is an Azumaya algebra over $C^{G}$, and an $H^{*}$-DeMeyer-Kanzaki Galois extension if $B$ is an $H^{*}$-Azumaya Galois extension and $V_{B}\left(B^{H}\right)=C$.

Let $P$ be a finitely generated and projective module over a commutative ring $R$. Then for a prime ideal $p$ of $R, P_{p}\left(=P \otimes_{R} R_{p}\right)$ is a free module over $R_{p}$ (= the local ring of $R$ at $p$ ), and the rank of $P_{p}$ over $R_{p}$ is the number of copies of $R_{p}$ in $P_{p}$, that is, $\operatorname{rank}_{R_{p}}\left(P_{p}\right)=m$ for some integer $m$. It is known that the $\operatorname{rank}_{R}(P)$ is a continuous function $\operatorname{(rank}_{R}(P)(p)=\operatorname{rank}_{R_{p}}\left(P_{p}\right)=m$ ) from $\operatorname{Spec}(R)$ to the set of nonnegative integers with the discrete topology (see [4, Corollary 4.11, page 31]). We will use the $\operatorname{rank}_{R}(P)$-function for a finitely generated and projective module $P$ over a commutative ring $R$.

3. $H^{*}$-Azumaya Galois extensions. In this section, keeping all notations as given in Section 2, we will characterize an $H^{*}$-Azumaya Galois extension $B$ in terms of the commutator $V_{B}\left(B^{H}\right)$ of $B^{H}$ in $B$.

THEOREM 3.1. If $B=B^{H} \cdot V_{B}\left(B^{H}\right)$, then $\left(V_{B}\left(B^{H}\right)\right)^{H}=C^{H}$.

Proof. Since $C \subset V_{B}\left(B^{H}\right), C^{H} \subset\left(V_{B}\left(B^{H}\right)\right)^{H}$. Conversely, since $V_{B}\left(B^{H}\right) \subset B$, $\left(V_{B}\left(B^{H}\right)\right)^{H} \subset B^{H}$. Hence $\left(V_{B}\left(B^{H}\right)\right)^{H} \subset B^{H} \cap V_{B}\left(B^{H}\right) \subset$ the center of $V_{B}\left(B^{H}\right)$. But $B=B^{H} \cdot V_{B}\left(B^{H}\right)$, so the center of $V_{B}\left(B^{H}\right)$ is $C$. Thus, $\left(V_{B}\left(B^{H}\right)\right)^{H} \subset C^{H}$.

THEOREM 3.2. A ring $B$ is an $H^{*}$-Azumaya Galois extension of $B^{H}$ if and only if $B=B^{H} \cdot V_{B}\left(B^{H}\right)$ such that $V_{B}\left(B^{H}\right)$ is an $H^{*}$-Azumaya Galois extension of $C^{H}$ and $B^{H}$ is an Azumaya $C^{H}$-algebra.

Proof. $\quad(\Rightarrow)$ Since $B$ is an $H^{*}$-Azumaya Galois extension of $B^{H}$, then $V_{B}\left(B^{H}\right)$ is an $H^{*}$-Azumaya Galois extension of $\left(V_{B}\left(B^{H}\right)\right)^{H}$ (see [7, Lemma 4.1]) and $B^{H}$ is an Azumaya $C^{H}$-algebra (see [7, Theorem 3.4]). Moreover, by the proof of [7, Lemma 4.1], $B \# H$ is an Azumaya $C^{H}$-algebra such that $B \# H \cong B^{H} \otimes_{C^{H}}$ $\left(V_{B}\left(B^{H}\right) \# H\right) \cong B^{H}\left(V_{B}\left(B^{H}\right) \# H\right)$, where $B^{H}$ and $V_{B}\left(B^{H}\right) \# H$ are Azumaya $C^{H}$ algebras. But $H$ is a finite-dimensional Hopf algebra over a field $k$, so $B \cong$ $B^{H} \otimes_{C^{H}} V_{B}\left(B^{H}\right)$ from the isomorphism $B \# H \cong B^{H} \otimes_{C^{H}}\left(V_{B}\left(B^{H}\right) \# H\right)$, and so $B=B^{H} \cdot V_{B}\left(B^{H}\right)$. Hence $\left(V_{B}\left(B^{H}\right)\right)^{H}=C^{H}$ by Theorem 3.1. Thus $V_{B}\left(B^{H}\right)$ is an $H^{*}$-Azumaya Galois $C^{H}$-algebra.

$(\Leftarrow)$ Since $V_{B}\left(B^{H}\right)$ is an $H^{*}$-Azumaya Galois algebra over $C^{H}, V_{B}\left(B^{H}\right) \# H$ is an Azumaya $C^{H}$-algebra [7, Theorem 3.4]. By hypothesis, $B^{H}$ is an Azumaya $C^{H}$ algebra, so $B^{H} \otimes_{C H}\left(V_{B}\left(B^{H}\right) \# H\right) \cong B^{H} V_{B}\left(B^{H}\right) \# H=B \# H$ which is an Azumaya 
$C^{H}$-algebra. Thus $B \# H$ is a Hirata separable extension of $B$ (see [5, Theorem 1]). Moreover, $V_{B}\left(B^{H}\right)$ is a separable $C^{H}$-algebra (see [7, Theorem 3.4]) and $B^{H}$ is an Azumaya $C^{H}$-algebra by hypothesis, so $B^{H} \cdot V_{B}\left(B^{H}\right)(=B)$ is also a separable $C^{H}$-algebra. Thus $B$ is an $H^{*}$-Azumaya Galois extension of $B^{H}$ [7, Theorem 3.4].

Next we generalize the characterization of a commutative Galois algebra as given by Ikehata (see [5, Theorem 2]) to a commutative $H^{*}$-Galois algebra.

LEMMA 3.3. If $C$ is a commutative $H^{*}$-Galois algebra over $C^{H}$, then $C$ is a maximal commutative subalgebra of $\mathrm{C} \# \mathrm{H}$.

Proof. Since $C$ is a commutative $H^{*}$-Galois algebra over $C^{H}, C \# H \cong$ $\operatorname{Hom}_{C^{H}}(C, C)$ [6, Theorem 1.7]. Hence it suffices to show that $V_{\mathrm{Hom}_{C H}(C, C)}\left(C_{L}\right)$ $=C_{L}$ where $C_{L}=\left\{c_{L}\right.$, the left multiplication map induced by $\left.c \in C\right\}$. In fact, $C_{L} \subset V_{\mathrm{Hom}_{C H}(C, C)}\left(C_{L}\right)$ is clear. Conversely, let $f \in V_{\mathrm{Hom}_{C H}(C, C)}\left(C_{L}\right)$. Then, for each $c \in C,(c f)(x)=(f c)(x)$ for all $x \in C$. Hence $c f(x)=f(c x)$, and so $c f(1)=f(c)$ for all $c \in C$. Thus $f(c)=d_{f}(c)$ for all $c \in C$, where $d_{f}=f(1) \in$ $C$, that is, $f=\left(d_{f}\right)_{L} \in C_{L}$.

THEOREM 3.4. Let $C$ be a commutative separable $C^{H}$-algebra containing $C^{H}$ as a direct summand as a $C^{H}$-module. Then, $C$ is a commutative $H^{*}$-Galois algebra over $C^{H}$ if and only if $C \otimes_{C^{H}}(C \# H) \cong M_{n}(C)$, the matrix algebra over $C$ of order $n$ where $n$ is the dimension of $H$ over $k$.

Proof. $(\Rightarrow)$ Since $C$ is an $H^{*}$-Galois algebra over $C^{H}, C \# H \cong \operatorname{Hom}_{C H}(C, C)$ such that $C$ is finitely generated and projective over $C^{H}$ [6, Theorem 1.7]. Hence $C \# H$ is an Azumaya $C^{H}$-algebra and $C$ is a maximal commutative subalgebra of the Azumaya $C^{H}$-algebra $C \# H$ by Lemma 3.3. By hypothesis, $C$ is also a separable $C^{H}$-algebra, so $C$ is a splitting ring for the Azumaya $C^{H}$-algebra $C \# H$ such that $C \otimes_{C^{H}}(C \# H) \cong \operatorname{Hom}_{C}(C \# H, C \# H)$ (see the proof of [4, Theorem 5.5, page 64]). Noting that $C \# H=C \otimes_{k} H$ which is a free $C$-module of rank $n$ where $n=\operatorname{dim}_{k}(H)$, we have that $C \otimes_{C^{H}}(C \# H) \cong M_{n}(C)$.

$(\Leftarrow)$ Since $C \otimes_{C^{H}}(C \# H) \cong M_{n}(C), C \otimes_{C^{H}}(C \# H)$ is an Azumaya $C$-algebra. By hypothesis, $C^{H}$ is a direct summand of $C$ as a $C^{H}$-module, so $C \# H$ is an Azumaya $C^{H}$-algebra [4, Corollary 1.10, page 45]. Hence $C \# H$ is a Hirata separable extension of $C$. But $C$ is a separable $C^{H}$-algebra by hypothesis, so $C$ is an $H^{*}$-Galois algebra over $C^{H}$ [7, Theorem 3.4].

We remark that the necessity does not need the hypothesis that $C^{H}$ is a direct summand of $C$.

4. $H^{*}$-DeMeyer-Kanzaki Galois extensions. We recall that $B$ is an $H^{*}$ DeMeyer-Kanzaki Galois extension of $B^{H}$ if $B$ is an $H^{*}$-Azumaya Galois extension of $B^{H}$ and $V_{B}\left(B^{H}\right)=C$. In this section, we characterize an $H^{*}$-DeMeyerKanzaki Galois extension in terms of the smash product $V_{B}\left(B^{H}\right) \# H$ and prove that $C$ is a splitting ring for the Azumaya $C^{H}$-algebras $V_{B}\left(B^{H}\right) \# H$ and $B \# H$. 
THeOrem 4.1. Let $B$ be an $H^{*}$-Azumaya Galois extension of $B^{H}$. Then the following statements are equivalent:

(1) $B$ is an $H^{*}$-DeMeyer-Kanzaki Galois extension of $B^{H}$;

(2) $\operatorname{rank}_{C^{H}}\left(V_{B}\left(B^{H}\right)\right)=\operatorname{rank}_{C^{H}}(C)$;

(3) $C$ is a maximal commutative separable subalgebra of $V_{B}\left(B^{H}\right) \# H$.

PROOF. $\quad(1) \Rightarrow(2)$. It is clear.

$(2) \Rightarrow(1)$. Since $B$ is an $H^{*}$-Azumaya Galois extension of $B^{H}, V_{B}\left(B^{H}\right)$ is an $H^{*}$-Azumaya Galois algebra over $C^{H}$ by Theorem 3.2 such that $V_{B}\left(B^{H}\right)$ is a separable and finitely generated projective module over $C^{H}$ (see [7, Theorem 3.4]). Hence the rank function $\operatorname{rank}_{C^{H}}\left(V_{B}\left(B^{H}\right)\right)$ is defined and $V_{B}\left(B^{H}\right)$ is an Azumaya algebra over its center [4, Theorem 3.8, page 55]. But $B=B^{H} \cdot V_{B}\left(B^{H}\right)$ by Theorem 3.2, so the center of $V_{B}\left(B^{H}\right)$ is $C$. Thus $V_{B}\left(B^{H}\right)$ is an Azumaya $C$ algebra; and so $C$ is a direct summand $V_{B}\left(B^{H}\right)$ as a $C$-module. This implies that $C$ is a direct summand $V_{B}\left(B^{H}\right)$ as a $C^{H}$-module. Therefore the rank function $\operatorname{rank}_{C^{H}}(C)$ is also defined. Now by hypothesis, $\operatorname{rank}_{C^{H}}\left(V_{B}\left(B^{H}\right)\right)=\operatorname{rank}_{C^{H}}(C)$, so $V_{B}\left(B^{H}\right)=C$, that is, $B$ is an $H^{*}$-DeMeyer-Kanzaki Galois extension of $B^{H}$.

$(1) \Rightarrow(3)$. Since $B$ is an $H^{*}$-DeMeyer-Kanzaki Galois extension of $B^{H}, B$ is an $H^{*}$-Azumaya Galois extension such that $V_{B}\left(B^{H}\right)=C$. Hence $B=B^{H} \cdot V_{B}\left(B^{H}\right) \cong$ $B^{H} \otimes_{C^{H}} C$ such that $C$ is an $H^{*}$-Galois algebra over $C^{H}$ by Theorem 3.2, and so $C$ is a separable $C^{H}$-algebra containing $C^{H}$ as a direct summand as a $C^{H}$-module [7, Theorem 3.4]. Hence $C$ is a maximal commutative separable subalgebra of $C \# H$ where $C=V_{B}\left(B^{H}\right)$ by Lemma 3.3.

$(3) \Rightarrow(2)$. Since $B$ is an $H^{*}$-Azumaya Galois extension of $B^{H}, B=B^{H} \cdot V_{B}\left(B^{H}\right) \cong$ $B^{H} \otimes_{C^{H}} V_{B}\left(B^{H}\right)$ such that $V_{B}\left(B^{H}\right)$ is an $H^{*}$-Azumaya Galois algebra over $C^{H}$ by Theorem 3.2. Hence $V_{B}\left(B^{H}\right) \# H$ is an Azumaya $C^{H}$-algebra and $V_{B}\left(B^{H}\right)$ is an Azumaya $C$-algebra [7, Theorem 3.4]. By hypothesis, $C$ is a maximal commutative separable subalgebra of $V_{B}\left(B^{H}\right) \# H$, so

$$
C \otimes_{C^{H}}\left(V_{B}\left(B^{H}\right) \# H\right) \cong \operatorname{Hom}_{C}\left(V_{B}\left(B^{H}\right) \# H, V_{B}\left(B^{H}\right) \# H\right)
$$

(see [4, Theorem 5.5, page 64]). On the other hand, $V_{B}\left(B^{H}\right) \# H \cong \operatorname{Hom}_{C}\left(V_{B}\left(B^{H}\right)\right.$, $V_{B}\left(B^{H}\right)$ ) (see [7, Theorem 3.4]). Thus

$$
\begin{aligned}
C \otimes_{C^{H}}\left(V_{B}\left(B^{H}\right) \# H\right) & \cong C \otimes_{C^{H}} \operatorname{Hom}_{C^{H}}\left(V_{B}\left(B^{H}\right), V_{B}\left(B^{H}\right)\right) \\
& \cong \operatorname{Hom}_{C}\left(C \otimes_{C^{H}} V_{B}\left(B^{H}\right), C \otimes_{C^{H}} V_{B}\left(B^{H}\right)\right) ;
\end{aligned}
$$

and so $\operatorname{Hom}_{C}\left(V_{B}\left(B^{H}\right) \# H, V_{B}\left(B^{H}\right) \# H\right) \cong \operatorname{Hom}_{C}\left(C \otimes_{C^{H}} V_{B}\left(B^{H}\right), C \otimes_{C^{H}} V_{B}\left(B^{H}\right)\right)$. This implies that $V_{B}\left(B^{H}\right) \# H \cong P \otimes_{C}\left(C \otimes_{C^{H}} V_{B}\left(B^{H}\right)\right)$ for some finitely generated projective $C$-module $P$ of rank 1 , that is, $V_{B}\left(B^{H}\right) \# H \cong P \otimes_{C^{H}} V_{B}\left(B^{H}\right)$. Taking $\operatorname{rank}_{C^{H}}()$ both sides, we have that $n \cdot \operatorname{rank}_{C^{H}}\left(V_{B}\left(B^{H}\right)\right)=\left(\operatorname{rank}_{C^{H}}(P)\right)$. $\operatorname{(rank}_{C^{H}}\left(V_{B}\left(B^{H}\right)\right)$ ) where $n=\operatorname{dim}_{k}(H)$. But $\operatorname{rank}_{C^{H}}\left(V_{B}\left(B^{H}\right)\right)$ is also $n$, so $\operatorname{rank}_{C^{H}}(C)=\operatorname{rank}_{C^{H}}(P)=n=\operatorname{rank}_{C^{H}}\left(V_{B}\left(B^{H}\right)\right)$. 
Theorem 4.1 implies that the Azumaya $C^{H}$-algebras $V_{B}\left(B^{H}\right) \# H$ and $B \# H$ have a nice splitting ring $C$ which is an $H^{*}$-Galois algebra over $C^{H}$ and separable over $C^{H}$ such that $C \otimes_{C^{H}}\left(V_{B}\left(B^{H}\right) \# H\right)$ and $C \otimes_{C^{H}}(B \# H)$ are matrix algebras.

COROLLARY 4.2. If $B$ is an $H^{*}$-DeMeyer-Kanzaki Galois extension of $B^{H}$, then $C \otimes_{C^{H}}\left(V_{B}\left(B^{H}\right) \# H\right) \cong M_{n}(C)$, the matrix algebra over $C$ of order $n$ where $n=$ $\operatorname{dim}_{k}(H)$.

Proof. By hypothesis, $B$ is an $H^{*}$-DeMeyer-Kanzaki Galois extension of $B^{H}$, so $C\left(=V_{B}\left(B^{H}\right)\right)$ is an $H^{*}$-Galois algebra over $C^{H}$ by Theorem 3.2. Hence $C$ is a separable $C^{H}$-algebra and $C \# H$ is an Azumaya $C^{H}$-algebra [7, Theorem 3.4]. Thus $C^{H}$ is a direct summand of $C$ as a $C^{H}$-module. Therefore, $C \otimes_{C^{H}}(C \# H) \cong$ $M_{n}(C)$ by Theorem 3.4.

COROLLARY 4.3. If $B$ is an $H^{*}$-DeMeyer-Kanzaki Galois extension of $B^{H}$, then $C \otimes_{C^{H}}(B \# H) \cong M_{n}(B)$, the matrix algebra over $B$ of order $n$ where $n=\operatorname{dim}_{k}(H)$.

Proof. By Corollary 4.2, $C \otimes_{C^{H}}(C \# H) \cong M_{n}(C)$, so

$$
B^{H} \otimes_{C^{H}} C \otimes_{C^{H}}(C \# H) \cong B^{H} \otimes_{C^{H}} M_{n}(C) .
$$

Since $B=B^{H} \cdot V_{B}\left(B^{H}\right) \cong B^{H} \otimes_{C^{H}} V_{B}\left(B^{H}\right)=B^{H} \otimes_{C^{H}} C$, we have that

$$
\begin{aligned}
C \otimes_{C^{H}}(B \# H) & \cong C \otimes_{C^{H}}\left(\left(B^{H} \otimes_{C^{H}} C\right) \# H\right) \\
& \cong C \otimes_{C^{H}} B^{H} \otimes_{C^{H}}(C \# H) \\
& \cong B^{H} \otimes_{C^{H}} C \otimes_{C^{H}}(C \# H) \\
& \cong B^{H} \otimes_{C^{H}} M_{n}(C) \cong M_{n}\left(B^{H} \otimes_{C^{H}} C\right) \\
& \cong M_{n}(B) .
\end{aligned}
$$

ACKNOWLEDGments. This paper was written under the support of a Caterpillar Fellowship at Bradley University. The authors would like to thank Caterpillar Inc. for the support.

\section{REFERENCES}

[1] R. Alfaro and G. Szeto, Skew group rings which are Azumaya, Comm. Algebra 23 (1995), no. 6, 2255-2261.

[2] _ On Galois extensions of an Azumaya algebra, Comm. Algebra 25 (1997), no. $6,1873-1882$.

[3] F. R. DeMeyer, Some notes on the general Galois theory of rings, Osaka J. Math. 2 (1965), 117-127.

[4] F. R. DeMeyer and E. Ingraham, Separable Algebras over Commutative Rings, Lecture Notes in Mathematics, vol. 181, Springer-Verlag, Berlin, 1971.

[5] S. Ikehata, Note on Azumaya algebras and H-separable extensions, Math. J. Okayama Univ. 23 (1981), no. 1, 17-18.

[6] H. F. Kreimer and M. Takeuchi, Hopf algebras and Galois extensions of an algebra, Indiana Univ. Math. J. 30 (1981), no. 5, 675-692.

[7] M. Ouyang, Azumaya extensions and Galois correspondence, Algebra Colloq. 7 (2000), no. 1, 43-57. 
[8] G. Szeto and L. Xue, Some correspondences for a center Galois extension, Math. Japon. 52 (2000), no. 3, 463-468.

[9] L. Xue, The invariant subrings of DeMeyer-Kanzaki Galois extensions, Port. Math. (N.S.) 59 (2002), no. 1, 37-45.

George Szeto: Department of Mathematics, Bradley University, Peoria, IL 61625, USA E-mail address: szeto@hi17top.brad1ey.edu

Lianyong Xue: Department of Mathematics, Bradley University, Peoria, IL 61625, USA E-mail address: 1xue@hi 17top.brad1ey.edu 


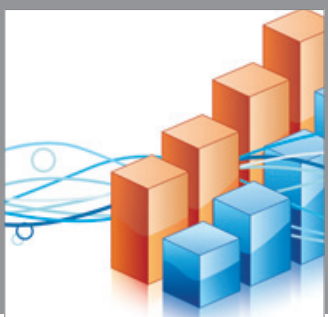

Advances in

Operations Research

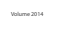

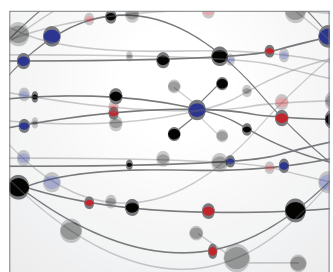

\section{The Scientific} World Journal
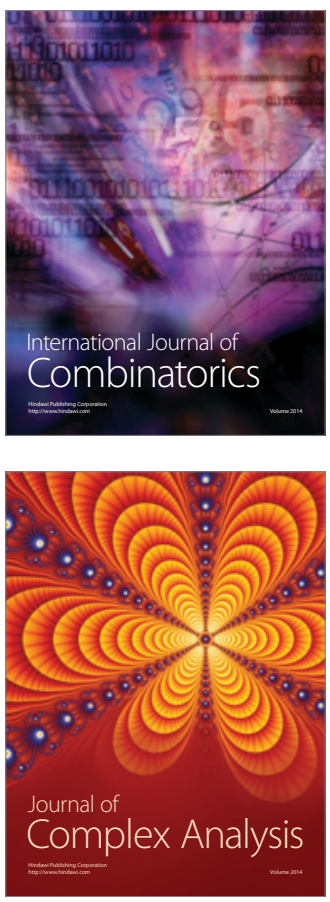

International Journal of

Mathematics and

Mathematical

Sciences
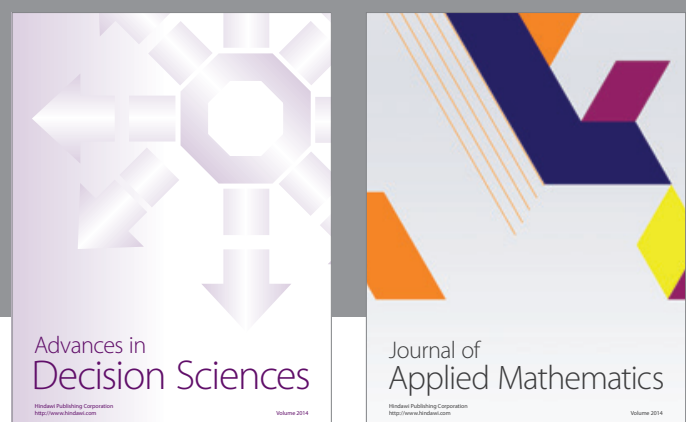

Journal of

Applied Mathematics
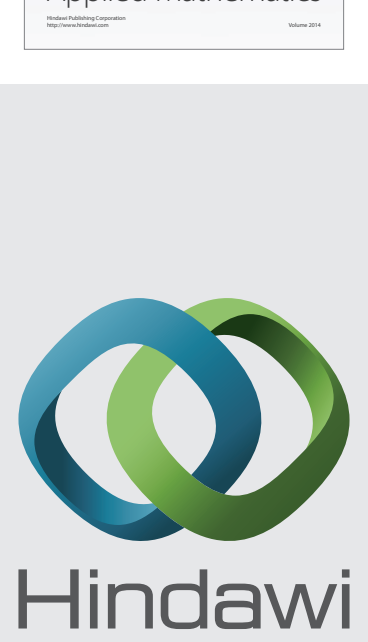

Submit your manuscripts at http://www.hindawi.com
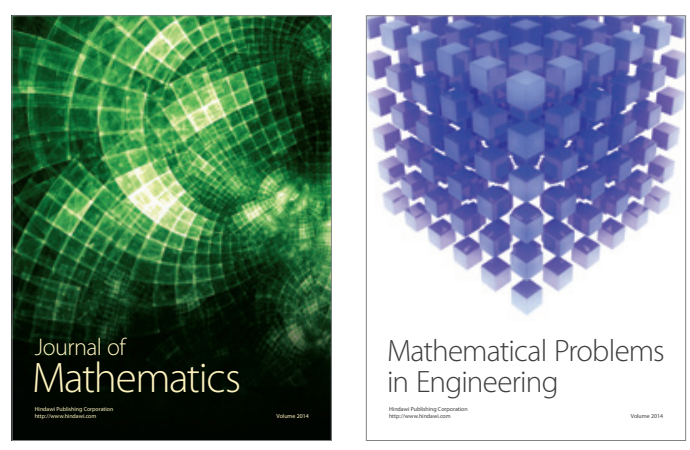

Mathematical Problems in Engineering
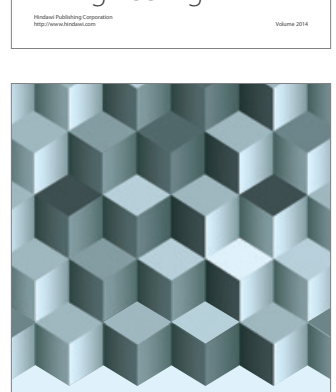

Journal of

Function Spaces
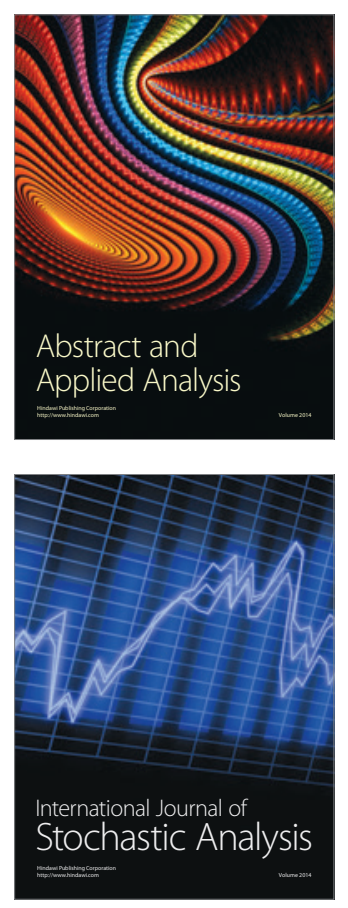

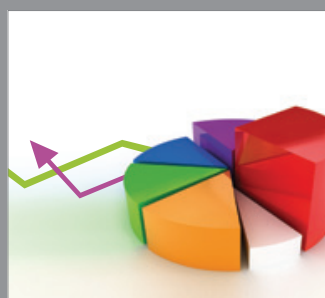

ournal of

Probability and Statistics

Promensencen
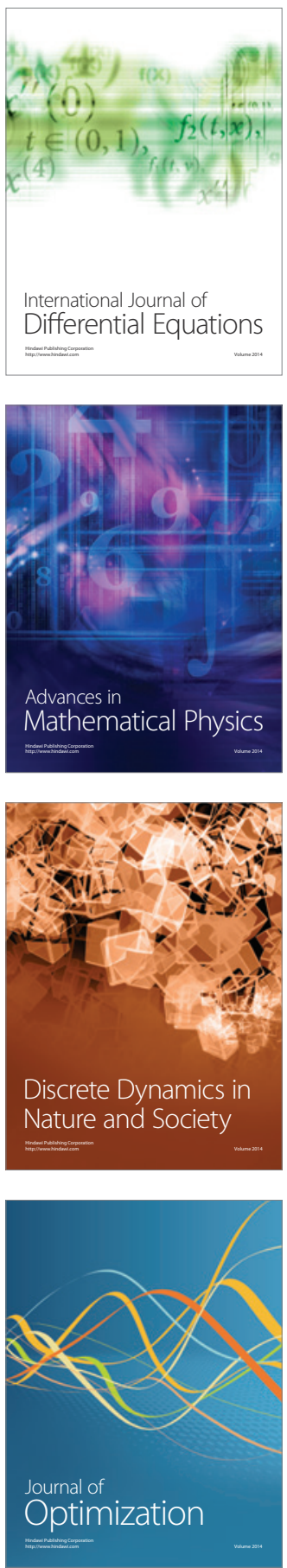